\title{
Design of Intelligent Traffic Monitoring System Based on MCU
}

\author{
Fu Xiuwei ${ }^{1, a,{ }^{,},}, \mathrm{Fu} \mathrm{Li}^{2, \mathrm{~b}}$ and Yang Muxi ${ }^{3, \mathrm{c}}$ \\ ${ }^{1}$ College of Information \& Control Engineering, Jilin Institute of Chemical Technology, Jilin 132022, \\ China \\ ${ }^{2}$ College of Information \& Control Engineering, Jilin Institute of Chemical Technology, Jilin 132022, \\ China \\ ${ }^{3}$ College of Information \& Control Engineering, Jilin Institute of Chemical Technology, Jilin 132022, \\ China \\ afxw7720268@163.com, bfuli247012412@126.com, c1711948553@qq.com \\ ${ }^{*}$ Corresponding author
}

Keywords: Flow, MCU, Flow Sensor, Monitoring.

\begin{abstract}
Mainly the hardware design of the traffic monitoring system is researched in this paper. The whole design includes selection of the turbine flow sensor, MCU peripheral circuits and the process for the software design. The collected traffic data through the mathematical model are analyzed and calculated in the system. The system that designed completely will be able to execute synchronously display and alarm of the measured flow, in order to achieve the purposes of the traffic monitoring.
\end{abstract}

\section{Introduction}

The design of flow monitoring system is to reduce the environmental pollution and reduce the loss of power consumption. It can measure liquid, gas or steam in different sites without flow media, and react directly to the monitoring center through data transmission to achieve the purpose of automatic detection and control of flow rate. Flow collection needs sensor, transmission and display need MCU and peripheral circuit. Therefore, based on the study and reference of monitoring system at home and abroad, this paper designs a set of single-chip microcomputer as the core, which can realize real-time monitoring of flow. Synchronous display of sewage flows monitoring system.

\section{Flow acquisition principle}

A turbine Flow meter is installed in the center of the pipeline, both ends of which are supported by bearings. When the fluid passes through the pipe, the turbine blade is impinged and the driving moment is generated, which makes the turbine to overcome the friction torque and the fluid resistance moment and produce rotation. Because the blade has conductive magnetism, it is in the magnetic field of the signal detector (consisting of permanent magnetic steel and coil). The rotating blade cuts the magnetic field line and periodically changes the magnetic flux of the coil, which induces the electric pulse signal at both ends of the coil. After amplifying and shaping the signal, a continuous rectangular pulse wave with a certain amplitude is formed, which can be transmitted far to the display instrument to show the instantaneous flow rate and accumulative amount of the fluid. In a certain flow range, the rotating angular velocity of the turbine is proportional to the flow velocity for a certain viscosity of the fluid medium. The frequency of the electric pulse signal is proportional to the volume flow of the fluid flowing through the turbine flow sensor. The formula is explained below. 


\section{Circuit design}

\subsection{Overall circuit design}

The flow integration system is the secondary instrument of the Flow meter, which can process and calculate the analog signal or pulse signal from the flow sensor and transmitter, and display and accumulate the calculated results from the display and integration unit, and have the flow retransmission function and the communication function. The flow integrator mainly includes microcontroller unit, signal front-end processing circuit, analog input and output channel, display circuit, serial communication interface circuit, alarm display circuit and clock circuit.

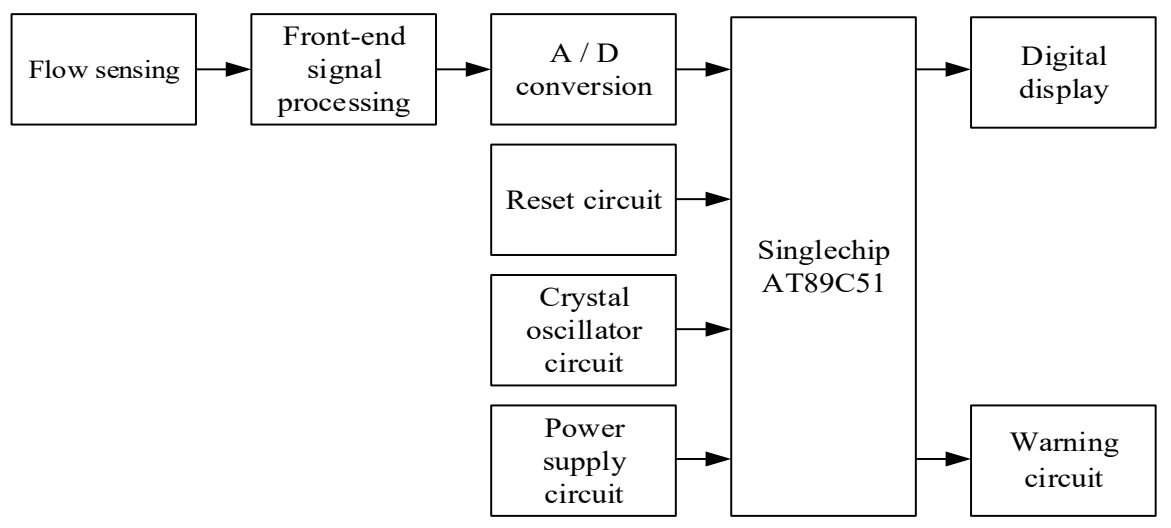

Fig. 1. block diagram of hardware structure for flow accumulator

The working principle is that the $4-20 \mathrm{~mA}$ standard analog signal sent by the flow sensor is controlled by the control system with single chip microcomputer as the core. The single chip microcomputer controls the opening and closing of its circuit, and the sensor connected to the analog switch sends the collected analog signal into the subsequent signal conversion circuit, converts the analog current signal into the voltage signal, and then sends the analog current signal to the single-chip computer system via A / D conversion. After processing with software such as square operation, scale operation and scale transformation according to the mathematical model, the actual flow value is obtained, and the accumulated integral flow is obtained, and the result is displayed on the display composed of LCD according to the requirement. An alarm is issued when the flow size exceeds the standard signal or reaches the minimum value.

\subsection{I / V conversion circuit}

A series of signal processing must be carried out before the analog signal from the sensor enters into the A / D conversion. The turbine Flow meter is the output signal of $4-20 \mathrm{~mA}$ current. In the measuring system, it is easy to measure and process the voltage, and the measuring precision is also. High, so it is often necessary to convert current or other forms of electrical signals into voltage signals. 


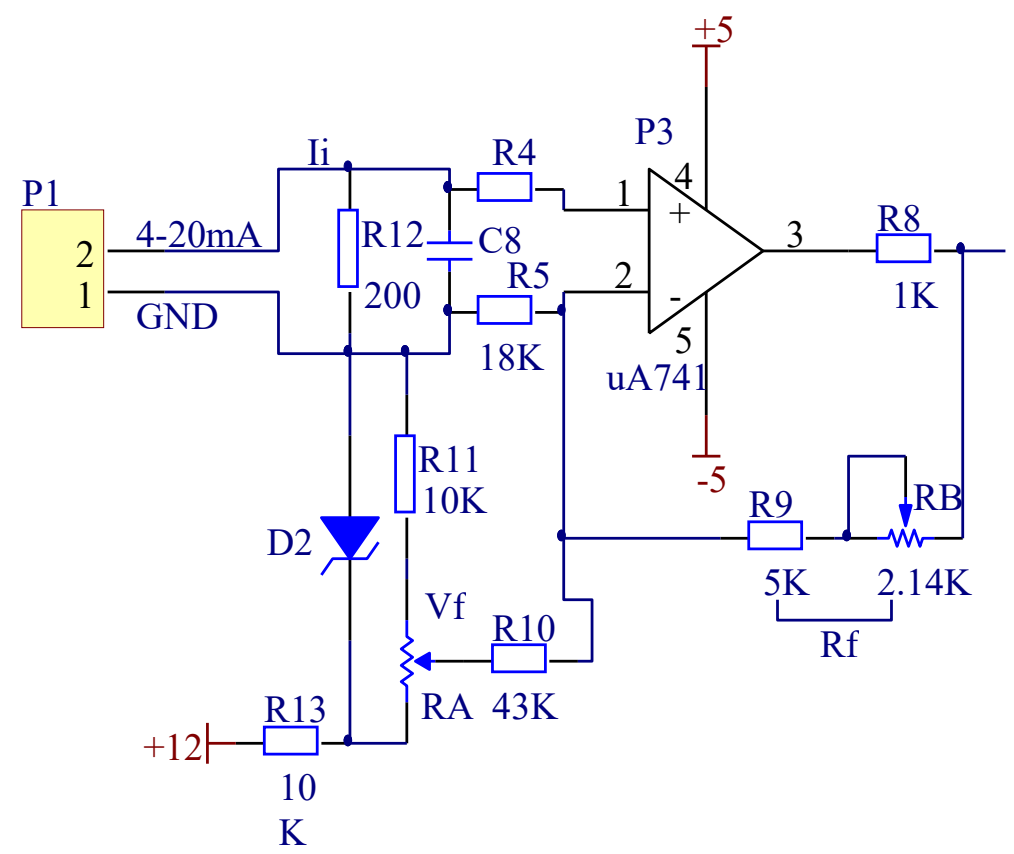

Fig. 2. I / V conversion circuit

For the above circuit analysis, it is shown that the current passing through the feedback resistor $\mathrm{Rf}$ is equal to that of $\mathrm{V}_{\mathrm{N}} / \mathrm{R}_{5}+\left(V_{N}-V_{f}\right) / R_{10}{ }^{[1]}$. The conversion formula is:

$$
V_{0}=\left(1+R_{f} / R_{5}+R_{f} / R_{10}\right) \cdot V_{N}-\left(R_{12} / R_{10}\right) \cdot V_{f}
$$

The $\mathrm{V}_{\mathrm{N}}$ in the upper formula can be replaced by $I_{\mathrm{i}} \times R_{4}$. If $\mathrm{R} 4=200 \Omega, \mathrm{R} 5=18 \mathrm{k} \Omega, \mathrm{Rf}=7.14 \mathrm{k} \Omega, \mathrm{R} 10=43 \mathrm{k} \Omega$, and $V_{f} \approx 7.53 \mathrm{~V}$, When input $4-20 \mathrm{~mA}$ current signal, corresponding output $0-5 \mathrm{~V}$ voltage signal.

\subsection{A / D conversion circuit}

In the part of the A / D conversion circuit, we use ADC0809 as a CMOS module with 8-bit A / D converter / 8-channel multiplexer and microprocessor compatible control logic. It is a successive approximation A / D converter, and can directly interface with the single chip.

The ADC0809 consists of an 8-bit analog switch, an address latch and decoder, an A / D converter and a tractate output latch. The multi-way switch can be used for 8 analog channels, allowing 8 analog input to be converted. The tractate output latch is used to latch A / D converted digital quantity. When the OE end is high, the converted data can be fetched from the tractate output latch .ADC0809 requires input analog: signal unipolarity, voltage range $0-5 \mathrm{~V}$, if the signal is too small, must be amplified

\subsection{Display system circuits}

In this system, due to the need to display standard flow, instantaneous flow and cumulative flow information, the traditional digital tube display mode will bring inconvenience to users. Therefore, LCD1602 liquid crystal display module can be selected. It has the advantages of friendly interface, low power consumption, beautiful appearance and rich display content.

\subsection{Other circuits}

Reset circuit: the reset of single chip microcomputer is realized by external circuit. In normal operation, if there are two high levels of machine cycle time on RST pin, it can cause system reset.

Crystal oscillator circuit: the crystal oscillator combines with the internal circuit of the single chip microcomputer to produce the clock frequency required by the single chip microcomputer. It provides the basic clock signal for the system. 
Alarm circuit: the alarm circuit uses buzzer, when the flow in the system exceeds the standard flow or reaches the lowest value, the alarm will send out the alarm signal.

\section{Mathematical model of flow monitoring}

(1).When the input signal is an analog signal with a linear relationship with the flow rate The instantaneous volume flow is:

$$
\begin{aligned}
& Q=Q_{\max } I / 10 \\
& Q=Q_{\max }(I-4) / 16 \quad(4-10 \mathrm{~mA})
\end{aligned}
$$

Among them: the design conditions for the maximum volume flow can be set according to needs. (2).When the input signal is an analog signal with an open relation to the flow rate. Instantaneous volume flow is:

$$
\begin{aligned}
& Q=Q_{\max } \sqrt{\frac{I}{10}} \quad(0-10 \mathrm{~mA}) \\
& Q=Q_{\max } \sqrt{\frac{I-4}{10}} \quad(4-20 \mathrm{~mA})
\end{aligned}
$$

(3).When the input is a pulse signal, Instantaneous volume flow is:

$$
Q=(F \times 3600) / K \quad\left(m^{3} / \mathrm{h}\right)
$$

$\mathrm{F}(1 / \mathrm{s})$ is pulse input signal frequency. $\mathrm{K}\left(1 / \mathrm{m}^{3}\right)$ is flow coefficient of pulse output flow meter. Pulse number is corresponding to unit volume when $\mathrm{Q}$ is volume flow.

Through the above formula, when the input is an analog current signal or pulse signal, the instantaneous flow rate can be calculated, and then the cumulative flow rate can be calculated by the relation between the accumulated flow rate and the instantaneous flow rate.

$$
Q^{\prime}=\frac{1}{K_{T}} \int q \frac{d t}{R_{i}}
$$

Where: $\mathrm{Q}^{\prime}$ is the cumulative flow value and dt is the sampling period and $\mathrm{KT}$ is the integral multiplier, and $\mathrm{Ri}$ is the conversion coefficient of instantaneous flow per unit time [3].

\section{Conclusion}

In this paper, the hardware part of the flow monitoring system is designed, and the I / V / A / D converter is used to realize the flow display and alarm function. This design structure is simple, economical and applicable, and has high superiority and feasibility. It can monitor the instantaneous and accumulative sewage discharge of the pollution source enterprise and the operation state of the enterprise sewage treatment facility at any time, and it can also exceed the standard alarm and so on. It is of great significance to control the enterprise sewage discharge.

\section{References}

[1] Tong Shibai, Hua Chengying. Fundamentals of Analog Electronic Technology [M]. Beijing: higher Education Press 2006.

[2] Zhang Yi Gang. Principle and Application of single Chip Microcomputer [M]. Higher Education Press. 2003.

[3] Wang Huiyi, Ma Minkang. Application of AT89C51 single Chip Microcomputer [J]. Industrial metrology 1997 / 1997: 06: 2-18. 